\title{
Enzymatic synthesis of bis-5-alkylresorcinols by resorcinol-producing type III polyketide synthases
}

\author{
Akimasa Miyanaga and Sueharu Horinouchi \\ No enzyme systems responsible for the biosynthesis of structurally and biosynthetically intriguing bis-5-alkylresorcinols produced \\ by plants have been identified. Herein, we show that bacterial, fungal and plant alkylresorcinol-producing type III polyketide \\ synthases (PKSs), such as ArsB in the Gram-negative bacterium Azotobacter vinelandii, ORAS in the fungus Neurospora crassa \\ and ARAS2 in the rice plant Oryza sativa, can synthesize bis-5-alkylresorcinol from alkanedioic acid $\mathrm{N}$-acetylcysteamine \\ dithioester as a starter substrate and from malonyl-CoA as an extender substrate by two-step conversion. Plants presumably use \\ a type III PKS for the biosynthesis of bis-5-alkylresorcinols.
}

The Journal of Antibiotics (2009) 62, 371-376; doi:10.1038/ja.2009.44; published online 26 June 2009

Keywords: bis-5-alkylresorcinol; enzymatic synthesis; polyketide synthase

\section{INTRODUCTION}

Type III polyketide synthases (PKSs) are structurally simple enzymes that catalyze the synthesis of aromatic polyketides in bacteria, fungi and plants. ${ }^{1}$ Type III PKSs catalyze iterative decarboxylative condensations of an extender substrate such as malonyl-CoA with a starter substrate such as acyl-CoA, and subsequently cyclize the resultant polyketide chain to yield various bioactive natural compounds with great structural diversity. This structural diversity of polyketide scaffolds is governed mainly by the selectivity of starter and extender substrates, the number of condensation reactions and the mode of ring closure of the resultant polyketide chains. One class of the products of type III PKSs includes alkylresorcinols that consist of polar dihydroxybenzene rings and hydrophobic alkyl chains, and exhibit a wide variety of biological and biochemical activities. ${ }^{2-6}$ For example, ArsB, a bacterial type III PKS from Azotobacter vinelandii, catalyzes three condensations of malonyl-CoA with a long-chain acyl starter substrate and subsequently cyclizes the resultant tetraketide intermediate by aldol condensation to yield alkylresorcinol. ${ }^{3,4}$ ORAS, a fungal type III PKS from Neurospora crassa, synthesizes tetraketide and pentaketide alkylresorcylic acids from a long-chain acyl starter substrate such as stearoyl-CoA. ${ }^{5}$ ARAS2 (Oryza sativa genome DB os10g08620, alkylresorcylic acid synthase 2), a plant type III PKS from the rice plant O. sativa, also produces a tetraketide alkylresorcylic acid from a longchain acyl starter substrate, as does ORAS (our unpublished data). The alkylresorcylic acids produced by ORAS and ARAS2 are immediately converted into alkylresorcinols by non-enzymatic decarboxylation.

Bis-5-alkylresorcinol, having a dihydroxybenzene ring at both terminal ends of an alkyl chain, has a relatively uncommon chemical structure (Figure 1a). The alkyl chain length of the bis-5-alkylresorcinols so far isolated, for instance, from Hakea trifurcata, ${ }^{7}$ Oncostemon bojeranum, ${ }^{8}$ Panopsis rubescens ${ }^{9}$ and the rye Secale cereale, ${ }^{10}$ ranges from C14 to C22. Some of the bis-5-alkylresorcinols contain one or two double bonds in their alkyl chains. Bis-5-alkylresorcinols were shown to be capable of both mediating $\mathrm{Cu}^{2+}$-dependent DNA cleavage and potentiating the action of bleomycin through the inhibition of DNA polymerase $\beta .{ }^{11}$ It seems plausible that these two activities could function synergistically to potentiate the destruction of cancer cells. A transient inhibition of DNA polymerase $\beta$, concomitant with anti-tumor therapy, improves the effectiveness of DNA-damaging anti-tumor agents. Although the chemical synthesis method has been developed because of the important biological activities of bis-5-alkylresorcinols, ${ }^{12}$ enzyme systems responsible for their biosynthesis have not yet been identified. As mentioned above, our recent studies of type III PKSs showed that a dihydroxybenzene ring of alkylresorcinols is derived from polyketide intermediate chains. We thus hypothesized that a type III PKS could synthesize bis-5alkylresorcinols. If so, as all the PKSs so far found in plants belong to the type III PKS family, bis-5-alkylresorcinols in plants must also be synthesized by a type III PKS(s). In this case, two reactions by a type III PKS(s) are required for the formation of two hydroxybenzene rings in bis-5-alkylresorcinol, as depicted in Figure 1b. In this proposed pathway, the acyl substrate used in the second ring formation is uncommon. It was obscure whether type III PKSs could accept long-chain acyl thioesters harboring an aromatic ring in the alkyl tail as a starter substrate in the second reaction. In this report, we describe that ArsB, ARAS2 and ORAS, all belonging to the type III PKS family, show the ability to synthesize bis-5-alkylresorcinol from alkanedioic acid $\mathrm{N}$-acetylcysteamine (NAC) dithioester as a starter substrate and from malonyl$\mathrm{CoA}$ as an extender substrate. 
a<smiles>Oc1cc(O)cc(CCCCCCCCCCCCCCCCc2cc(O)cc(O)c2)c1</smiles><smiles>Oc1cc(O)cc(CCCC=CCCCCCCCCCc2cc(O)cc(O)c2)c1</smiles>

bis-5-alkylresorcinol 14:1 from Panopsis rubescens<smiles>Oc1cc(O)cc(CCCCCC=CCC=CCCCCCCCCc2cc(O)cc(O)c2)c1</smiles>

bis-5-alkylresorcinol 16:2 from Secale cereale

b

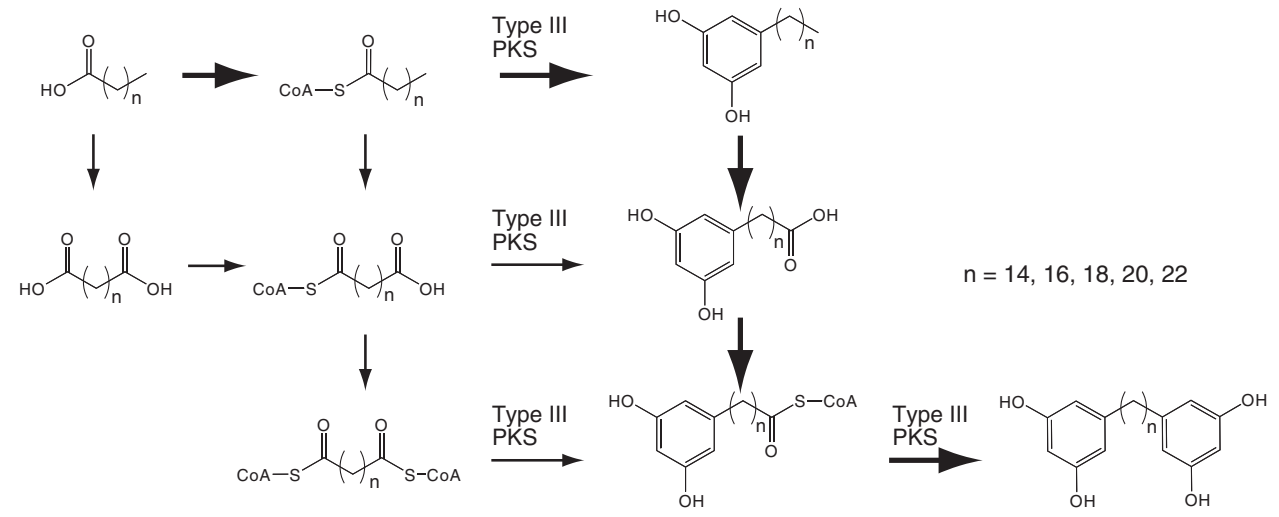

c

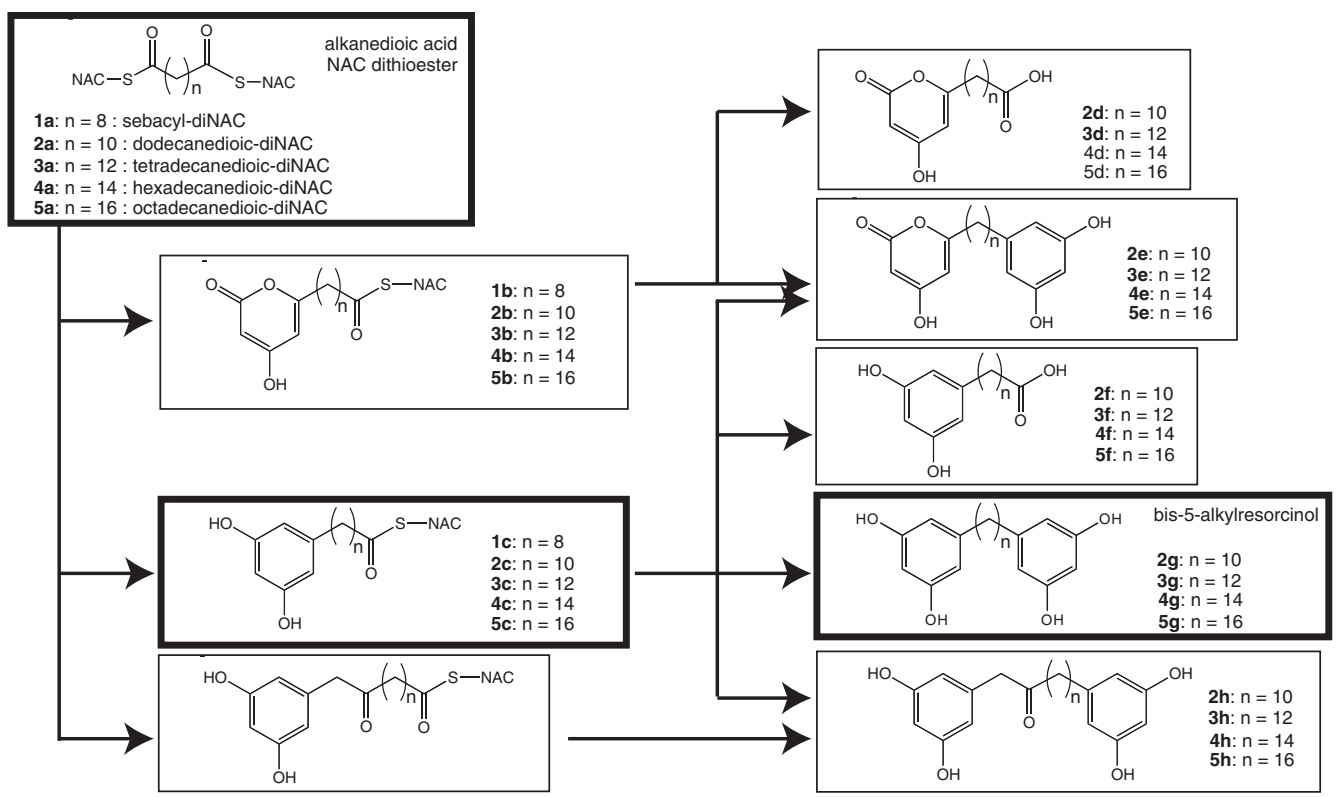

Figure 1 Bis-5-alkylresorcinol synthesis. (a) A variety of natural bis-5-alkylresorcinols. (b) A proposed biosynthetic pathway of bis-5-alkylresorcinol in plants. (c) A proposed synthetic route of bis-5-alkylresorcinols and by-products by type III polyketide synthases (PKSs) from alkanedioic acid NAC (Nacetylcysteamine) dithioester substrates in vitro. A part of the proposed synthetic route of bis-5-alkylresorcinols by type III PKSs is highlighted by using thicker squares.

\section{MATERIALS AND METHODS}

\section{Materials}

The carboxylic acids used were purchased from Tokyo Chemical Industry (Tokyo, Japan) and from Apollo Scientific Limited (Bredbury,
UK). The NAC compounds used in this study were chemically synthesized from their corresponding carboxylic acids according to a published method. ${ }^{13}$ Recombinant ArsB, ARAS2 and ORAS were prepared, as described. 3,5 


\section{Reaction of type III PKS with NAC compound}

Recombinant type III PKS $(2.0 \mu \mathrm{M})$ was incubated at $30^{\circ} \mathrm{C}$ for $6 \mathrm{~h}$ with $50 \mu \mathrm{M}$ NAC compound, $100 \mu \mathrm{M}$ malonyl-CoA, $20 \mathrm{~mm}$ HEPES-Na (pH 7.5), $150 \mathrm{~mm} \mathrm{NaCl}$ and $10 \%(\mathrm{w} / \mathrm{v})$ glycerol in a total volume of $200 \mu \mathrm{l}$. After incubation at $30^{\circ} \mathrm{C}$ for $6 \mathrm{~h}$, the reactions were quenched by the addition of $20 \mu \mathrm{l}$ of $6 \mathrm{M} \mathrm{HCl}$. The products were extracted with $400 \mu \mathrm{l}$ of ethyl acetate, and the organic layer was evaporated to dryness. The residual material was dissolved in methanol for HPLC, liquid chromatography-atmospheric pressure chemical ionization mass spectrometry (LC-APCI/MS) and radio-TLC analyses. HPLC and LC-APCI/MS analyses were carried out using an esquire high-capacity trap plus system (Bruker Daltonics, Bremen, Germany) and a Hitachi LaChrom ELITE system (Hitachi, Tokyo, Japan), respectively, on the apparatus equipped with a Pegasil-B C4 reversed-phase column $(4 \times 250 \mathrm{~mm}$; Senshu Scientific, Tokyo, Japan). The products were eluted with 55 or $70 \%$ acetonitrile in water containing $0.1 \%$ trifluoroacetic acid as an eluent at a flow rate of $1 \mathrm{ml} \mathrm{min}^{-1}$. For radio-TLC analysis, a silica-gel $60 \mathrm{WF}_{254}$ TLC plate (Merck, Darmstadt, Germany) was developed in benzene/acetone/acetic acid (75:25:1, $\mathrm{v} / \mathrm{v} / \mathrm{v})$ and the ${ }^{14} \mathrm{C}$-labeled compounds derived from the substrate $\left[2-{ }^{14} \mathrm{C}\right] \mathrm{mal}-$ onyl-CoA were detected using a Fujifilm BAS-MS imaging plate (Fujifilm, Tokyo, Japan). The identification of ${ }^{14} \mathrm{C}$-labeled signals on TLC was confirmed by the co-migration of radioactivity with each product purified by HPLC.

\section{${ }^{1} \mathrm{H}$ NMR spectral data of synthetic compounds}

Sebacyl-diNAC (1a): H NMR $\left(500 \mathrm{MHz}, \mathrm{CDCl}_{3}\right) \delta 5.85$ (2H, br), 3.44 (4H, dd, $J=12.5,6 \mathrm{~Hz}), 3.03(4 \mathrm{H}, \mathrm{t}, J=6 \mathrm{~Hz}), 2.58(4 \mathrm{H}, \mathrm{t}, J=7.5 \mathrm{~Hz}), 1.97(6 \mathrm{H}, \mathrm{s}), 1.66$ $(4 \mathrm{H}, \mathrm{t}, J=7.5 \mathrm{~Hz}), 1.27(8 \mathrm{H}, \mathrm{m})$.

Dodecanedioyl-diNAC (2a): H NMR $\left(500 \mathrm{MHz}, \mathrm{CDCl}_{3}\right) \delta 5.86(2 \mathrm{H}, \mathrm{br})$, $3.44(4 \mathrm{H}, \mathrm{dd}, J=12.5,6.5 \mathrm{~Hz}), 3.03(4 \mathrm{H}, \mathrm{t}, J=6.5 \mathrm{~Hz}), 2.58(4 \mathrm{H}, \mathrm{t}, J=7.5 \mathrm{~Hz})$, $1.97(6 \mathrm{H}, \mathrm{s}), 1.66(4 \mathrm{H}, \mathrm{t}, J=7.5 \mathrm{~Hz}), 1.28(12 \mathrm{H}, \mathrm{m})$.

Tetradecanedioyl-diNAC (3a): H NMR $\left(500 \mathrm{MHz}, \mathrm{CDCl}_{3}\right) \delta 5.84(2 \mathrm{H}, \mathrm{br})$, $3.44(4 \mathrm{H}, \mathrm{dd}, J=12.5,6.5 \mathrm{~Hz}), 3.03(4 \mathrm{H}, \mathrm{t}, J=6.5 \mathrm{~Hz}), 2.58(4 \mathrm{H}, \mathrm{t}, J=8 \mathrm{~Hz}), 1.97$ $(6 \mathrm{H}, \mathrm{s}), 1.66(4 \mathrm{H}, \mathrm{t}, J=7.5 \mathrm{~Hz}), 1.26(16 \mathrm{H}, \mathrm{m})$.

Hexadecanedioyl-diNAC (4a): H NMR $\left(500 \mathrm{MHz}, \mathrm{CDCl}_{3}\right) \delta 5.85(2 \mathrm{H}, \mathrm{br})$, $3.44(4 \mathrm{H}, \mathrm{dd}, J=12.5,6 \mathrm{~Hz}), 3.03(4 \mathrm{H}, \mathrm{t}, J=6 \mathrm{~Hz}), 2.58(4 \mathrm{H}, \mathrm{t}, J=7.5 \mathrm{~Hz}), 1.98$ $(6 \mathrm{H}, \mathrm{s}), 1.66(4 \mathrm{H}, \mathrm{t}, J=7.5 \mathrm{~Hz}), 1.26(20 \mathrm{H}, \mathrm{m})$.

1,3-Dihydroxy-5-(14'-(3", $5^{\prime \prime}$-dihydroxyphenyl)tetradecenyl)benzene $\quad(\mathbf{4 g})$ : H NMR $\left(500 \mathrm{MHz}\right.$, THF- $\left.d_{8}\right) \delta 7.79(4 \mathrm{H}, \mathrm{s}), 6.03(4 \mathrm{H}, \mathrm{d}, J=2 \mathrm{~Hz}), 5.97$ $(2 \mathrm{H}, \mathrm{t}, J=2 \mathrm{~Hz}), 2.41(4 \mathrm{H}, \mathrm{t}, J=8 \mathrm{~Hz}), 1.60(4 \mathrm{H}, \mathrm{br}), 1.29(20 \mathrm{H}, \mathrm{m})$.

Octadecanedioyl-diNAC (5a): H NMR $\left(500 \mathrm{MHz}, \mathrm{CDCl}_{3}\right) \delta 5.83(2 \mathrm{H}, \mathrm{s})$, $3.45(4 \mathrm{H}, \mathrm{dd}, J=12.5,6 \mathrm{~Hz}), 3.03(4 \mathrm{H}, \mathrm{t}, J=6.5 \mathrm{~Hz}), 2.58(4 \mathrm{H}, \mathrm{t}, J=7.5 \mathrm{~Hz}), 1.98$ $(6 \mathrm{H}, \mathrm{s}), 1.66(4 \mathrm{H}, \mathrm{t}, J=7.5 \mathrm{~Hz}), 1.26(24 \mathrm{H}, \mathrm{m})$.

15-Phenylpentadecanoyl-NAC (6c): H NMR $\left(500 \mathrm{MHz}, \mathrm{CDCl}_{3}\right) \delta 7.28(2 \mathrm{H}$, t, $J=7.5 \mathrm{~Hz}), 7.18(3 \mathrm{H}, \mathrm{m}), 3.44(2 \mathrm{H}, \mathrm{dd}, J=12.5,6 \mathrm{~Hz}), 3.03(2 \mathrm{H}, \mathrm{t}, J=6 \mathrm{~Hz})$, $2.60(2 \mathrm{H}, \mathrm{t}), 2.58(2 \mathrm{H}, \mathrm{t}), 1.98(3 \mathrm{H}, \mathrm{s}), 1.66(2 \mathrm{H}, \mathrm{t}, J=7.5 \mathrm{~Hz}), 1.61(2 \mathrm{H}, \mathrm{t}$, $J=7.5 \mathrm{~Hz}), 1.26(20 \mathrm{H}, \mathrm{m})$.

5-(14'-( $3^{\prime \prime}, 5^{\prime \prime}$-Dihydroxyphenyl)tetradecenyl)benzene (6g): H NMR $(500 \mathrm{MHz}$, THF- $\left.d_{8}\right) \delta 7.78(2 \mathrm{H}, \mathrm{s}), 7.20(2 \mathrm{H}, \mathrm{t}, J=7.5 \mathrm{~Hz}), 7.14(2 \mathrm{H}, \mathrm{d}, J=7 \mathrm{~Hz}), 7.10(\mathrm{H}, \mathrm{t}$, $J=7 \mathrm{~Hz}), 6.03(2 \mathrm{H}, \mathrm{d}, J=2 \mathrm{~Hz}), 5.97(\mathrm{H}, \mathrm{t}, J=2 \mathrm{~Hz}), 2.58(2 \mathrm{H}, \mathrm{t}, J=8 \mathrm{~Hz}), 2.41$ $(2 \mathrm{H}, \mathrm{t}, J=8 \mathrm{~Hz}), 1.60(4 \mathrm{H}, \mathrm{m}), 1.29(20 \mathrm{H}, \mathrm{m})$.

\section{Spectrometric data}

Sebacyl-diNAC (1a): HPLC ( $45 \%$ acetonitrile): $R_{\mathrm{t}}=9.0$, LC-APCI/MS (positive): MS, $m / z 405[\mathrm{M}+\mathrm{H}]^{+}$, UV: $\lambda_{\max } 231 \mathrm{~nm}$.

Dodecanedioyl-diNAC (2a): HPLC ( $45 \%$ acetonitrile): $R_{\mathrm{t}}=12.5$, LC-APCI/ MS (positive): MS, $m / z 433[\mathrm{M}+\mathrm{H}]^{+}$, UV: $\lambda_{\max } 231 \mathrm{~nm}$.

Tetradecanedioyl-diNAC (3a): HPLC ( $50 \%$ acetonitrile): $R_{\mathrm{t}}=13.0$, LC-APCI/

MS (positive): MS, $m / z 461[\mathrm{M}+\mathrm{H}]^{+}$, UV: $\lambda_{\max } 231 \mathrm{~nm}$.

Hexadecanedioyl-diNAC (4a): HPLC ( $55 \%$ acetonitrile): $R_{\mathrm{t}}=13.4$, LC-APCI/

MS (positive): MS, $m / z 489[\mathrm{M}+\mathrm{H}]^{+}$, UV: $\lambda_{\max } 231 \mathrm{~nm}$.

Compound 4c: HPLC (55\% acetonitrile): $R_{\mathrm{t}}=11.7$, LC-APCI/MS (positive): MS, $m / z 452[\mathrm{M}+\mathrm{H}]^{+}$.

Compound 4d: HPLC (55\% acetonitrile): $R_{\mathrm{t}}=7.2$, LC-APCI/MS (negative): MS, $m / z 351[\mathrm{M}-\mathrm{H}]^{-}$, UV: $\lambda_{\max } 285 \mathrm{~nm}$.

Compound 4e: HPLC (55\% acetonitrile): $R_{\mathrm{t}}=8.9$, LC-APCI/MS (negative): MS, $m / z 415[\mathrm{M}-\mathrm{H}]^{-}$, UV: $\lambda_{\max } 282 \mathrm{~nm}$.
Compound 4f: HPLC (55\% acetonitrile): $R_{\mathrm{t}}=8.3$, LC-APCI/MS (negative): MS, $m / z 349[\mathrm{M}-\mathrm{H}]^{-}$, MS/MS (precursor ion at $m / z 349$ ), $m / z$ 331, 287, UV: $\lambda_{\max } 274 \mathrm{~nm}$.

1,3-Dihydroxy-5-(14'-(3", $5^{\prime \prime}$-dihydroxyphenyl)tetradecenyl)benzene (4g): HPLC (55\% acetonitrile): $R_{\mathrm{t}}=9.9$, LC-APCI/MS (negative): MS, $\mathrm{m} / \mathrm{z} 413$ $[\mathrm{M}-\mathrm{H}]^{-}, \mathrm{MS} / \mathrm{MS}$ (precursor ion at $m / z 413$ ), $m / z 369$, UV: $\lambda_{\max } 274 \mathrm{~nm}$.

Compound 4h: HPLC ( $55 \%$ acetonitrile): $R_{\mathrm{t}}=7.8$, LC-APCI/MS (negative): MS, $m / z 455[\mathrm{M}-\mathrm{H}]^{-}$, UV: $\lambda_{\max } 274 \mathrm{~nm}$.

Tetradecanedioyl-diNAC (5a): HPLC ( $60 \%$ acetonitrile): $R_{\mathrm{t}}=13.6$, LC-APCI/ MS (positive): MS, $m / z 517[\mathrm{M}+\mathrm{H}]^{+}$, UV: $\lambda_{\max } 231 \mathrm{~nm}$.

15-Phenylpentadecanoyl-NAC (6c): HPLC (70\% acetonitrile): $R_{\mathrm{t}}=15.9$, LC-APCI/MS (positive): MS, $m / z 420[\mathrm{M}+\mathrm{H}]^{+}$, UV: $\lambda_{\max } 231 \mathrm{~nm}$.

5-(14'-(3", $5^{\prime \prime}$-Dihydroxyphenyl)tetradecenyl)benzene $(\mathbf{6 g})$ : HPLC $(70 \%$ acetonitrile): $R_{\mathrm{t}}=11.8$, LC-APCI/MS (negative): MS, $m / z 381[\mathrm{M}-\mathrm{H}]^{-}$, MS/MS (precursor ion at $m / z 381$ ), $m / z 339$, UV: $\lambda_{\max } 274 \mathrm{~nm}$.

\section{RESULTS}

\section{Reaction of ArsB with alkanedioic acid NAC dithioesters}

To investigate whether a type III PKS has the ability to synthesize bis-5-alkylresorcinol, we synthesized hexadecanedioyl-diNAC (4a) as an analog of hexadecanedioyl-diCoA, which is one of the candidates for the initial substrate. When ArsB was incubated with $\mathbf{4 a}$ and malonyl-CoA, two compounds in a major peak $4 \mathbf{g}$ and a minor peak 4e were detected in HPLC analysis (Figure 2b). Neither of the two compounds was detected in the control experiments performed with a boiled enzyme (Figure 2a). The molecular mass of $\mathbf{4 g}$ was 414 , on the basis of the $[\mathrm{M}-\mathrm{H}]^{-}$ion at $m / z 413$ on LC-APCI/MS analysis. To determine the chemical structure of $\mathbf{4 g}$ by ${ }^{1} \mathrm{H}$ NMR spectroscopy, we obtained $\mathbf{4 g}(0.3 \mathrm{mg}, 7 \%$ yield $)$ from a large-scale enzyme reaction. As a result, $\mathbf{4 g}$ was identified as 1,3-dihydroxy-5$\left(14^{\prime}-\left(3^{\prime \prime}, 5^{\prime \prime}\right.\right.$-dihydroxyphenyl)tetradecenyl)benzene by comparing its ${ }^{1} \mathrm{H}$ NMR spectrum with that of the synthetic compound in the previous report. ${ }^{12} \mathrm{~A}$ minor peak $4 \mathrm{e}$ was predicted to be a by-product, which possessed both a resorcinol and a pyrone group, on the basis of LC-APCI/MS analysis.

As shown in Figure 1c, in this reaction, ArsB might first convert one of the two NAC groups of 4 a into a resorcinol group, yielding an intermediate (4c). ArsB might subsequently accept $4 c$ as a substrate in the second reaction and convert the remaining NAC group of $4 \mathrm{c}$ into a resorcinol group, yielding bis-5-alkylresorcinol $(\mathbf{4 g})$. To clarify this prediction, we examined the course of the production of $4 \mathrm{c}$ and $4 \mathrm{~g}$ from 4a. During the early stage of the reaction with ArsB, for about 5-10 min after the reaction initiation, $4 \mathbf{c}$ peak was detected by the LC-APCI/MS analysis, concomitant with the reduction in the amount of $4 \mathbf{a}$ (Figures $2 \mathrm{e}-\mathrm{g}$ ), suggesting that $\mathbf{4 a}$ was converted to $4 \mathbf{c}$. Thereafter, the $\mathbf{4 g}$ peak was gradually increased, concomitant with the reduction in the amount of $\mathbf{4 c}$, suggesting that $4 \mathbf{c}$ was converted into $\mathbf{4 g}$. Such a two-step process in the production of $\mathbf{4 g}$ hampered us in determining the kinetic parameters of ArsB. The $\mathbf{4} \mathbf{g}$ production by ArsB is similar to the curcuminoid production by CUS (curcuminoid synthase) from $O$. sativa ${ }^{14}$ in terms of the two-step conversion. However, the mechanism of curcuminoid synthesis is different from that of bis-5-alkylresorcinol synthesis. CUS uses same starter substrates and two different extender substrates in the curcuminoid synthesis, whereas ArsB uses two different starter substrates and the same extender substrate in the bis-5-alkylresorcinol synthesis.

To investigate whether ArsB is able to accept an acyl thioester harboring an aromatic ring, we synthesized 15-phenylpentadecanoylNAC (6c) as a substrate analog in the second reaction. When ArsB was incubated with $\mathbf{6 c}$ and malonyl-CoA, a single compound $(\mathbf{6 g})$ was produced (Figures 3 and 4). This compound was predicted as 

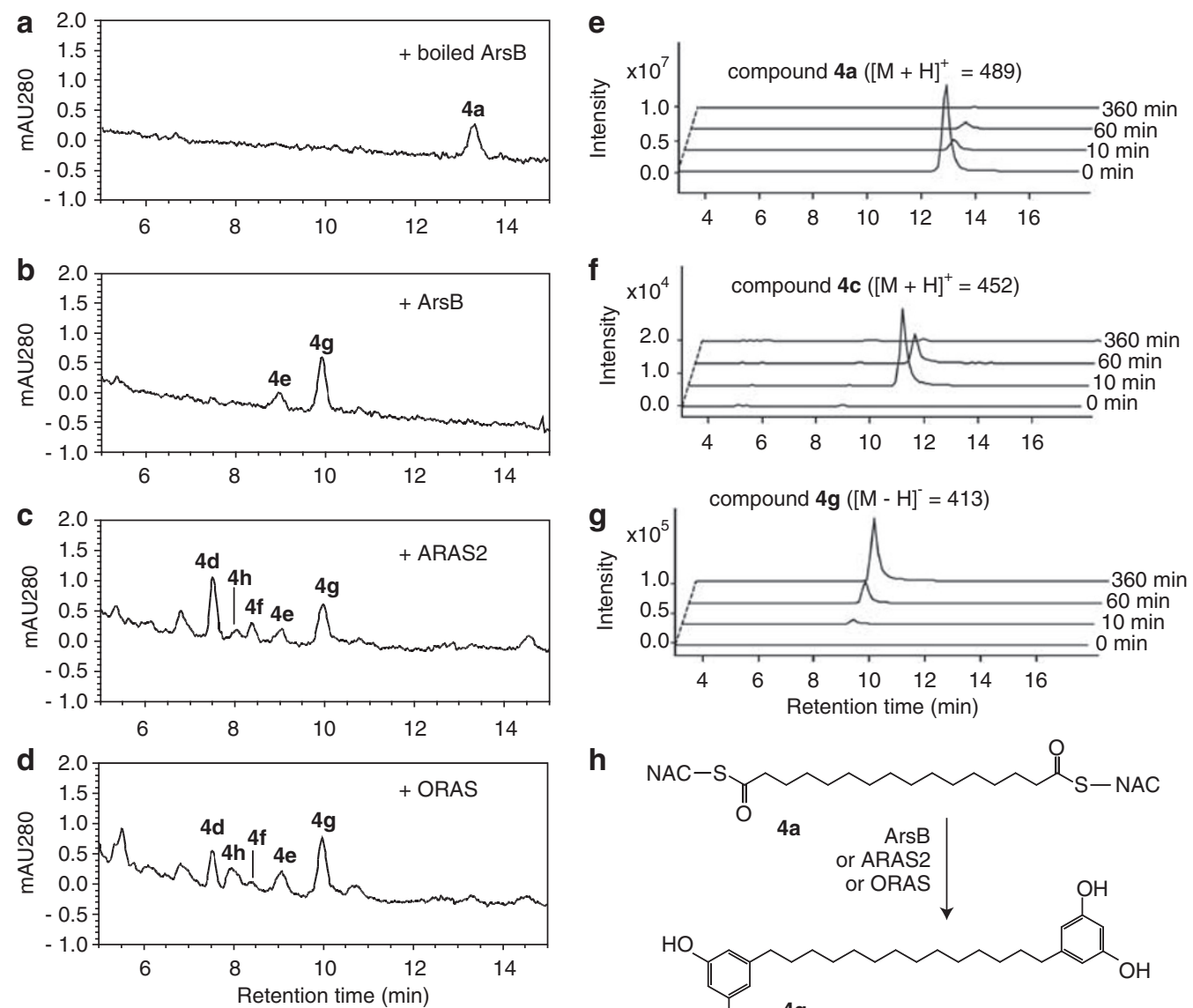

h

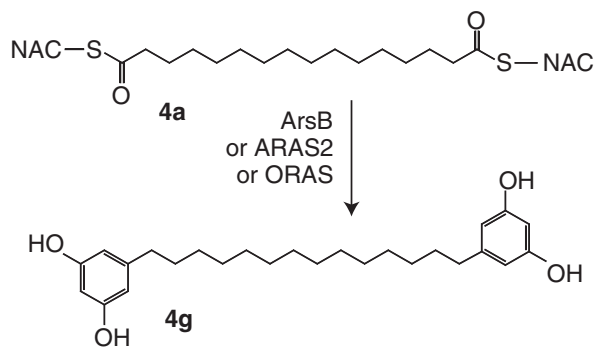

Figure 2 Chromatography analysis of the in vitro products of type III polyketide synthases (PKSs). (a, b, $\mathbf{c}$ and $\mathbf{d}$ ) HPLC chromatograms of the in vitro reactions of type III PKSs with 4a and malonyl-CoA. The reaction mixture contained boiled ArsB as a negative control (a), ArsB (b), ARAS2 (c) and ORAS (d). (e, $\mathbf{f}$ and $\mathbf{g}$ ) Selected extracted ion LC-APCI/MS chromatograms of the in vitro reaction of ArsB with $\mathbf{4 a}$ and malonyl-CoA at four time points. These chromatograms were prepared at $\mathrm{m} / \mathrm{z} 489[\mathrm{M}+\mathrm{H}]^{+}(\mathbf{e}), \mathrm{m} / \mathrm{z} 452[\mathrm{M}+\mathrm{H}]^{+}(\mathrm{F})$ and $\mathrm{m} / \mathrm{z} 413[\mathrm{M}-\mathrm{H}]^{-}(\mathrm{g})$. The conversion of 4 a to $4 \mathrm{~g}$ catalyzed by these enzymes is shown (h).

$5-\left(14^{\prime}-\left(3^{\prime \prime}, 5^{\prime \prime}\right.\right.$-dihydroxyphenyl)tetradecenyl)benzene on the basis of LC-APCI/MS analysis. These observations clearly showed that ArsB could accept the acyl substrate possessing an aromatic ring at the alkyl tail as a substrate.

Substrate specificity of ArsB was determined by incubating four different, synthesized alkanedioic acid NAC dithioesters (1a, 2a, 3a and 5a) possessing various lengths of alkyl chains with ArsB and malonyl-CoA. As a result, the radio-TLC, HPLC and LC-APCI/MS analyses of the resultant products revealed a broad range of bis-5-alkylresorcinol productivities of ArsB. ArsB accepted dodecanedioyl-diNAC (2a), tetradecanedioyl-diNAC (3a) and octadecanedioyldiNAC (5a) as substrates and gave bis-5-alkylresorcinol compounds $(\mathbf{2 g}, \mathbf{3 g}$ and $\mathbf{5 g}$ ), as observed in the reaction of ArsB with $4 \mathrm{a}$ (Figure 4). In the reaction with $2 \mathrm{a}$ and $3 \mathrm{a}$, the intermediate compounds $(\mathbf{2 c}$ and 3c) were simultaneously produced. ArsB also accepted sebacyl-diNAC (1a) as a substrate, but gave only an intermediate compound (1c) (Figure 4). In the reaction of ArsB with 1a, only one of the two NAC groups in 1a was converted into a resorcinol and the other NAC group was retained, probably because ArsB could not carry out the second reaction. ArsB was thus revealed to exhibit broad substrate specificity for alkyl-diNAC substrates. These results show that ArsB can synthesize bis-5-alkylresorcinol.

\section{Reaction of other type III PKSs with alkanedioic acid NAC dithioesters}

We assayed other resorcinol-producing type III PKSs, such as ARAS2 and ORAS, by using $\mathbf{4 a}$ and malonyl-CoA as substrates. As expected, both enzymes synthesized $\mathbf{4 g}$, as observed in the reaction of ArsB (Figures $2 c$, $d$ and 4 ). However, the production of $\mathbf{4 g}$ by these enzymes was less effective than that by ArsB. This may be because of the production of by-products in significant amounts. The production of a by-product $4 \mathrm{e}$ was observed in both reactions, as in the ArsB reaction. Moreover, the production of other by-products $(\mathbf{4 d}, \mathbf{4 f}$ and $4 \mathrm{~h}$ ) was detected. Peak $4 \mathbf{d}$ was predicted to be a compound that possessed both a pyrone and a carboxyl group on the basis of LC-APCI/MS analysis. This carboxyl group may be produced by cleavage of the thioester bond between a carboxylic acid and NAC. Similarly, peak $\mathbf{4 f}$ was predicted to be a compound that possessed both a resorcinol and a carboxyl group. Peak $4 \mathrm{~h}$ was predicted to be a compound that possessed both a resorcinol and a 2 -oxoresorcinol group on the basis of LC-APCI/MS analysis. ArsB almost completely produces alkylresorcinols from long-chain acyl substrate, ${ }^{3}$ whereas ARAS2 and ORAS produce significant amounts of alkylpyrones, in addition to alkylresorcylic acids and alkylresorcinols. ${ }^{5}$ This property of ARAS2 and ORAS may cause a low productivity of $\mathbf{4 g}$ and a 

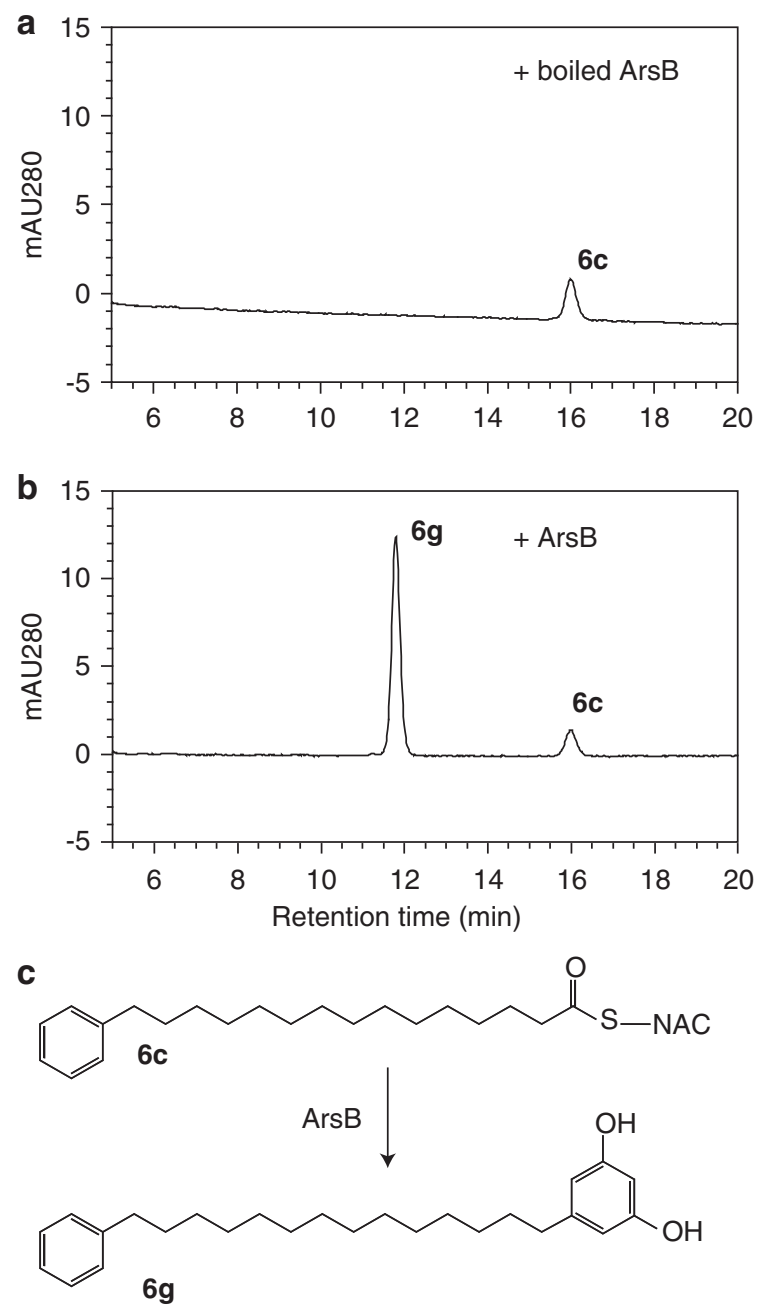

Figure 3 HPLC chromatograms of in vitro reactions of ArsB with $6 c$ and malonyl-CoA. The reaction mixture contained boiled ArsB as a negative control (a) and ArsB (b). The conversion of $6 \mathrm{c}$ to $6 \mathrm{~g}$ catalyzed by ArsB is shown (c).

by-product production in significant amounts. ARAS2 and ORAS were shown to produce alkylresorcylic acid from acyl-CoA. ${ }^{5}$ This fact permits us to speculate that ARAS2 and ORAS also synthesize resorcylic acid compounds as a direct product in this reaction. However, we could detect no resorcylic acid compound. This may be caused by the low productivity. Almost all of the resorcylic compounds produced by ARAS2 and ORAS in this reaction might have been converted into resorcinol compounds by non-enzymatic decarboxylation before the accumulation of resorcylic compounds. ARAS2 and ORAS also accepted $\mathbf{2 a}, \mathbf{3} \mathbf{a}$ and $\mathbf{5} \mathbf{a}$ as a substrate and gave bis-5-alkylresorcinol compounds ( $2 \mathrm{~g}, \mathbf{3 g}$ and $\mathbf{5 g}$ ) (data not shown). This substrate specificity is similar to that of ArsB. In addition, ARAS2 and ORAS accepted $\mathbf{6 c}$ as a substrate. When they were incubated with $\mathbf{6 c}$ and malonyl-CoA, $\mathbf{6 g}$ was mainly produced, although a by-product possessing a pyrone moiety was also produced (data not shown). This shows that ARAS2 and ORAS, similar to ArsB, can accept an acyl thioester harboring an aromatic ring as a substrate. These results show that ARAS2, a plant enzyme, and ORAS, a fungal enzyme, can synthesize bis-5-alkylresorcinol, as does ArsB, a bacterial enzyme.

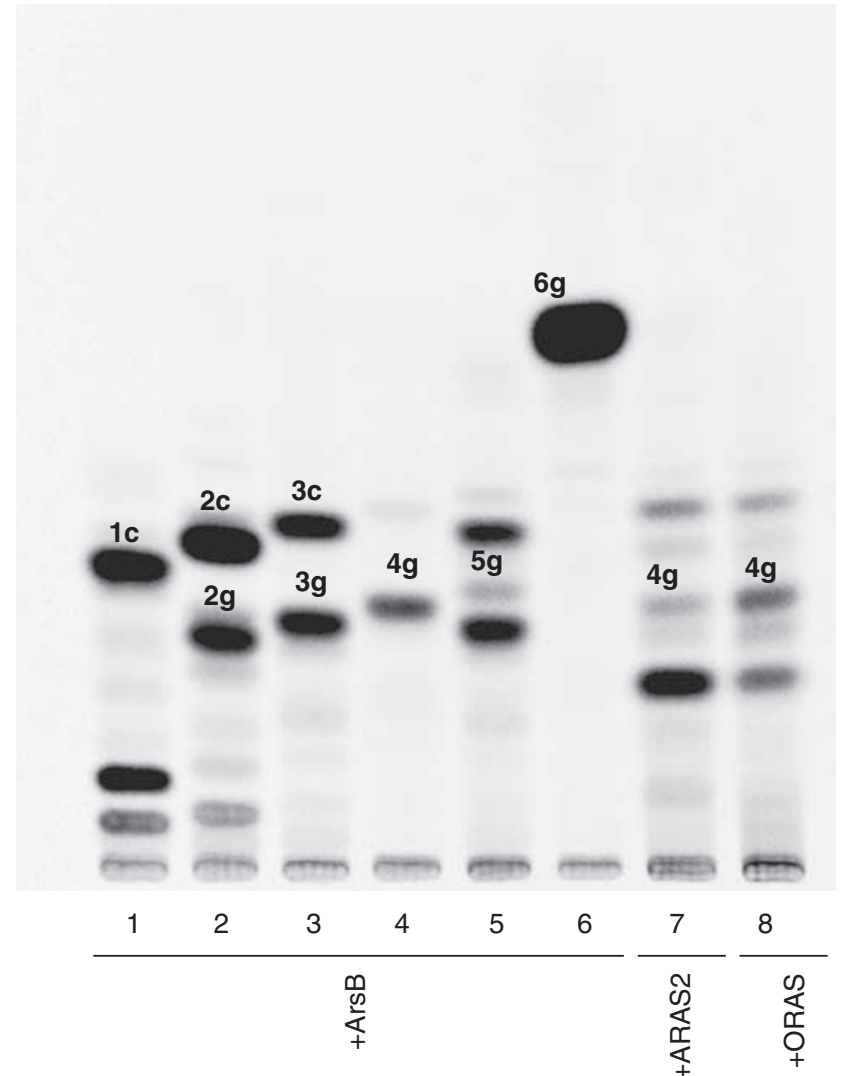

Figure 4 Radio-TLC analysis of products synthesized by type III polyketide synthases (PKSs) from various starter substrates and malonyl-CoA. The type III PKSs used were ArsB (lanes 1-6), ARAS2 (lane 7) and ORAS (lane 8). The starter substrates used were 1a (lane 1), 2a (lane 2), 3a (lane 3), 4a (lanes 4, 7 and 8), 5a (lane 5) and 6c (lane 6).

\section{DISCUSSION}

No type III PKS reactions against long-chain acyl thioesters harboring a bulkier substituent moiety at the alkyl tail have been reported. Our present data suggest that alkylresorcinol-producing type III PKSs can generally accept such unusual acyl thioesters as starter substrates. The crystal structures of type III PKSs revealed that some type III PKSs have an acyl-binding tunnel that accommodates a long acyl chain, extending from the active site to the surface of the protein. ${ }^{15,16}$ On the other hand, other type III PKSs such as chalcone synthases have no acyl-binding tunnel. The acyl-binding tunnel may be characteristic of all type III PKSs that use a long-chain acyl-CoA ester as a starter substrate. However, this tunnel seems to be too narrow for acyl substrates possessing bulkier substituents to accommodate whole molecules because of steric hindrance. This conflicts with our result that three type III PKSs could accept such acyl substrates. How do they accept such unusual acyl substrates? One speculation is that the alkyl chain of substrates bound in the acyl-binding tunnel is long enough to penetrate into the surface of the protein and the bulky aromatic group extrudes outside of the protein. However, this speculation can probably not be applied to type III PKSs. For example, ORAS perfectly envelopes an entire molecule of eicosanoic acid in its acyl-binding tunnel and the hydrocarbon tail of eicosanoic acid does not reach the surface of ORAS, as revealed by the X-ray crystallography of an ORAS-eicosanoic acid complex. ${ }^{16}$ PKS18 was also reported to hold a myristic acid molecule perfectly in its acyl-binding tunnel. ${ }^{17}$ Thus, 
the alkyl chain of substrate must be accommodated in the tunnel and must not penetrate into the surface of type III PKS.

Another speculation is that, during the reaction, some type III PKSs change the conformation of their acyl-binding tunnel so as to accommodate aromatic moiety. This hypothesis is supported by the crystal structures of ORAS. ORAS was reported to rearrange its conformation to accommodate eicosanoic acid into the acyl-binding tunnel on the basis of a comparison of the structures of the unliganded ORAS and an ORAS-eicosanoic acid complex. ${ }^{16}$ Some type III PKSs may thus acquire broad substrate specificity owing to the flexibility of the acyl-binding tunnel region. A short-chain acyl substrate 1a was not converted into bis-5-alkylresorcinol, although mono-alkylresorcinol intermediate $1 \mathrm{c}$ was produced. This observation suggests that type III PKSs cannot accommodate the aromatic ring attached to short-chain acyl intermediates, such as 1c, in the acylbinding tunnel.

Given that some type III PKSs, including a plant type III PKS, ARAS2, can synthesize bis-5-alkylresorcinols, we suppose that plants use a type III PKS for their biosynthesis. However, no type III PKS enzymes involved in the biosynthesis of bis-5-alkylresorcinol in plants have been isolated or predicted. The rye was reported to contain two type III PKS genes. ${ }^{17}$ These type III PKSs might be involved in the biosynthesis of bis-5-alkylresorcinols, although they are rather similar to chalcone synthases but not to alkylresorcinol-producing-type PKSs. We assume that alkylresorcinol-producing-type PKSs are involved in their biosynthesis in the rye plant, although they have not yet been identified.

In addition, the other part of the biosynthesis of bis-5-alkylresorcinol remains unclear. At least two enzymes, an oxidase and a CoAligase, are presumably necessary for the biosynthesis of bis-5-alkylresorcinol, other than the PKS reaction. One or more oxidases may have a function in the conversion of the methyl group at the alkyl tail into a carboxyl group, and CoA-ligase may have a function in the ligation of CoA to the resultant carboxyl group. A number of possible bis-5alkylresorcinol biosynthetic pathways including these enzymes are illustrated in Figure 1b. The most possible pathway is that bis-5alkylresorcinols are synthesized by alkylresorcinols, which are known to be distributed widely in plants. ${ }^{2}$ In this pathway, alkylresorcinols are first produced from acyl-CoAs. Next, the $\omega$-carbon of the alkyl chain of alkylresorcinols is oxidized, and the CoA is attached to the newly formed carboxyl group. Finally the resultant unusual acyl-CoA esters are converted into bis-5-alkylresorcinols by a type III PKS. The reaction in the final step is supported by our in vitro study that type III PKSs accepted unusual acyl thioesters as a substrate. Our present findings show that a type III PKS can produce a wide variety of bis-5-alkylresorcinol, the alkyl chain lengths of which are over C10. However, the alkyl chain lengths of bis-5-alkylresorcinols isolated from plants are limited to over C14. The step of producing substrates for type III PKSs may be controlled by the substrate specificity of the putative oxidase and CoA-ligase. Bis-5-alkylresorcinol has not been isolated from bacteria, fungi and rice plant. This may be caused by the lack of oxidase and ligase involved in the biosynthesis of bis-5alkylresorcinol in those organisms. Although further analysis is absolutely necessary to elucidate the biosynthetic pathway of bis-5alkylresorcinol in plants, we believe that a type III PKS is responsible for the final step in which it converts acyl-CoA esters into bis-5alkylresorcinols.

\section{ACKNOWLEDGEMENTS}

This work was supported by a research grant from the New Energy and Industrial Technology Development Organization of Japan and a Grant-in-Aid for Scientific Research on Priority Area 'Applied Genomics' from Monkasho.

1 Austin, M. B. \& Noel, J. P. The chalcone synthase superfamily of type III polyketide synthases. Nat. Prod. Rep. 20, 79-110 (2003).

2 Kozubek, A. \& Tyman, J. H. Resorcinolic lipids, the natural non-isoprenoid phenolic amphiphiles and their biological activity. Chem. Rev. 99, 1-26 (1999).

3 Funa, N., Ozawa, H., Hirata, A. \& Horinouchi, S. Phenolic lipid synthesis by type III polyketide synthases is essential for cyst formation in Azotobacter vinelandii. Proc. Natl. Acad. Sci. USA 103, 6356-6361 (2006).

4 Miyanaga, A., Funa, N., Awakawa, T. \& Horinouchi, S. Direct transfer of starter substrates from type I fatty acid synthase to type III polyketide synthases in phenolic lipid synthesis. Proc. Natl. Acad. Sci. USA 105, 871-876 (2008).

5 Funa, N., Awakawa, T. \& Horinouchi, S. Pentaketide resorcylic acid synthesis by type III polyketide synthase from Neurospora crassa. J. Biol. Chem. 282, 14476-14481 (2007).

6 Funabashi, M., Funa, N. \& Horinouchi, S. Phenolic lipids synthesized by type III polyketide synthase confer penicillin resistance on Streptomyces griseus. J. Biol. Chem. 283, 13983-13991 (2008).

7 Lytollis, W. et al. 5-Alkylresorcinols from Hakea trifurcata that cleave DNA. J. Am. Chem. Soc. 117, 12683-12690 (1995).

8 Chaturvedula, V. S. et al. New cytotoxic bis-5-alkylresorcinol derivatives from the leaves of Oncostemon bojerianum from the Madagascar rainforest. J. Nat. Prod. 65, 1627-1632 (2002).

9 Deng, J. Z., Starck, S. R. \& Hecht, S. M. Bis-5-alkylresorcinols from Panopsis rubescens that inhibit DNA polymerase $\beta$. J. Nat. Prod. 62, 477-480 (1999).

10 Suzuki, Y., Esumi, Y. \& Yamaguchi, I. Structures of 5-alkylresorcinol-related analogues in rye. Phytochemistry 52, 281-289 (1999).

11 Starck, S. R., Deng, J. Z. \& Hecht, S. M. Naturally occurring alkylresorcinols that mediate DNA damage and inhibit its repair. Biochemistry 39, 2413-2419 (2000).

12 Fürstner, A. \& Seidel, G. Shortcut syntheses of naturally occurring 5-alkylresorcinols with DNA-cleaving properties. J. Org. Chem. 62, 2332-2336 (1997).

13 Gilbert, I. H. et al. Synthesis of $\beta$-keto and $\alpha, \beta$-unsaturated $N$-acetylcysteamine thioesters. Bioorg. Med. Chem. Lett. 5, 1587-1590 (1995).

14 Katsuyama, Y., Matsuzawa, M., Funa, N. \& Horinouchi, S. In vitro synthesis of curcuminoids by type III polyketide synthase from Oryza sativa. J. Biol. Chem. 282, 37702-37709 (2007).

15 Sankaranarayanan, R. et al. A novel tunnel in mycobacterial type III polyketide synthase reveals the structural basis for generating diverse metabolites. Nat. Struct. Mol. Biol. 11, 894-900 (2004).

16 Rubin-Pitel, S. B. et al. Distinct structural elements dictate the specificity of the type III pentaketide synthase from Neurospora crassa. Chem. Biol. 15, 1079-1090 (2008).

17 Haussühl, K., Rohde, W. \& Weissenböeck, G. Expression of chalcone synthase genes in coleoptiles and primary leaves of Secale cereale L. after induction by UV radiation: evidence for a UV-protective role of the coleoptile. Bot. Acta. 109, 229-238 (1996). 